\title{
Affective Synchrony Predicts the Groupwork Performance of Children: Applying the Happiness Impression Scale to Measure Affective Accuracy on a Design Workshop
}

$1 \quad$ Rodrigo Fernandes ${ }^{1}$, Toshimasa Yamanaka ${ }^{2}$

$2{ }^{1}$ Laboratory of Kansei Sciences, Department of Kansei Behavioral and Brain Sciences, University of

3 Tsukuba, Tsukuba, Japan

$4 \quad{ }^{2}$ Laboratory X, Institute X, Department X, Organization X, City X, State XX (only USA, Canada and

5 Australia), Country

6 * Correspondence:

7 Rodrigo Fernandes

8 rqk.fernandes@gmail.com

9 Keywords: cooperation, children, motivation, affection, empathy, Synchrony, psychometrical

10 scales, socio-affection.

\section{Abstract}

12 Cooperative behavior develops throughout childhood when conditioned to variables such as empathy and affection. In group situations, Affective Synchronization - AS happens when participants accurately identify the feelings of their group members, creating a sense of synchrony. In this paper, we hypothesized that AS is a measurable indicator of children's group integration and group performance. We conducted a correlational study through a one-week design workshop to observe if higher AS levels would relate to higher group performance. 36 participants aged 6 to 14 y/o joined this workshop and 22 participants had their empathy levels evaluated as control. We developed and tested the Happiness Impression Scale - HIS to evaluate the AS level of participants after performing different workshop tasks. By the end of the workshop, participants performed group presentations which were scored by a jury and then correlated to their AS scores. Through the HIS scale, it was possible to establish differences between the AS levels of all groups. At a significant level, groups in which participants displayed higher levels of AS also received better evaluations from the jury. The combination between a participant being highly empathic and having high AS levels indicated to strongly affect their group performance. The measurement of AS levels indicated to be a stronger predictor of groupwork performance than traditional survey methods such as task and group satisfaction reports. Further studies should investigate which conditions might lead to higher AS levels, observe effects on immediate group behavior, and its implications on the development of cooperation throughout childhood.

\section{$30 \quad 1 \quad$ Introduction}

Cooperation, the act of working together in pursuit of a common goal, is a key factor for facing the increasing changes of our societies (Fehr \& Fischbacher, 2004). In order to cooperate, it is often necessary to take actions that does not directly benefit the individual but benefit the overall development of a group. Promoting cooperation is still a common challenge, as individuals can display different motivations to cooperate according to the to their personal values and to the given 
context. Thus, it is essential to consider how cooperation is motivated among individuals (Broadhead, 37 2003).

Childhood is a key period for the development of cooperation (Hepach, 2017; Warneken, 2015). In middle childhood (usually 6 to 12y/o), children are actively interacting with peers while exposed to formalized cooperation lessons (Council, 1984). According to Murata \& Yamaguchi (Murata, 2010), Japanese students in the first and secondary elementary school grades are usually required to take 34 hours per year of moral education and 100 hours of living environment studies. Even with the existence of formalized lesson, proper cooperative levels are not always established among children. Furthermore, much is unclear on how children differently assimilate cooperation when working together (Olson \& Spelke, 2008).

The term "Conditional Cooperation" describes how people's willingness to cooperate can vary according to contextual variables (Fischbacher, Gachter, \& Fehr, 2001). These variables can be: the familiarity level one has with the surrounding environment; the required level of cooperation in a task, or; the existing relationship with the people who require cooperation (Ezaki, Horita, Takezawa, \& Masuda, 2016; Fischbacher et al., 2001; Majolo \& Marechal, 2017). According to Lergetporer et al (Lergetporer, Angerer, Glätzle-Rützler, \& Sutter, 2014), individuals are more likely to cooperate with someone if they know that person is also willing to cooperate back. Further elaborating this, Angerer et al (Angerer, Glatzle-Rutzler, Lergetporer, \& Sutter, 2016), related cooperation to the levels of intimacy shared between individuals. Individuals are more inclined to cooperate with those perceived as closer, which implies that affectional bonds can be strong influencers of cooperation.

"Affect" is the experience of feeling and expressing emotions. affection and emotions have a big influence on children's motivations, facilitating their communication, attachment, and internalization (Roberts, 2018). Affectional bond is a type of attachment behavior that one individual has for another or, for example, how close or distant they feel about each other (Branscombe \& Wann, 1992). This is related to in-group/out-group feelings which leads individuals to perceive others as strongly connected or as distant strangers (Branscombe \& Wann, 1992). According to Angerer et al (Angerer et al., 2016), children of young age can already display prejudice bias when cooperating with individuals of different social or cultural background. These individuals can be perceived as too distant from their inner circles. On the other hand, individuals who have strong bonds can often share a state of synchrony where communication is facilitated, and cooperation is self-motivated (Vanutelli, Gatti, Angioletti, \& Balconi, 2017).

States of synchrony can be observed both on the physiological level, synchronizing hearts beats, electrical neuron activity, and respiratory patterns (Bernardi et al., 2009; Ellamil, Berson, \& Margulies, 2016; Ellamil, Berson, Wong, Buckley, \& Margulies, 2016; Khalil, Minces, McLoughlin, \& Chiba, 2013); and on the psychological level, sharing similar feelings and opinions about a task or subject (Asher \& Collins, 2012; Delaherche et al., 2012; Kuhn et al., 2011; Tschacher, Rees, \& Ramseyer, 2014). According to Vanutelli et al (2017), Affective Synchrony - AS, or the act of matching emotions with those of peers is positively related to increases in cooperation level among adult participants. However, much is unknown on the mechanisms and possible effects of this synchrony on group performance (Asher \& Collins, 2012; Delaherche et al., 2012; Neumann, Chan, Boyle, Wang, \& Westbury, 2015). Especially with children, there is a lack of tools and approaches to evaluate their AS levels (Neumann et al., 2015).

Like affectional bond, empathy is strongly related with cooperative behavior (Roberts, 2018). Empathy is the individual capability to detect and comprehend the feelings of others in different 
contexts (Tong et al., 2012). The difference between Empathy and Affective Synchrony is that while empathy is considered an innate individual capacity, it is possible to feel affectively synchronized with someone regardless of it. Rather, AS is likely to be affected by different contextual situations. As a counterpoint, new definitions on empathy are also describing it as situational and interactive, changing according to the involved people and situation (Tong et al., 2012). Like Affective Synchrony, there are only a few numbers of dedicate tools and research investigating the development of empathy on children (Neumann et al., 2015), but they are great indicators of social skills. Higher levels of empathic capacity are often related to higher levels of cooperative behavior (Roberts, 2018).

Different socio-affective strategies can be established from the development of empathy or the promotion of Affective Synchrony (Hendrix, van Herk, Verhaegh, \& Markopoulos, 2009). Basing on them, we considered the followings as possible motivating mechanisms for cooperative group behavior on children in the middle childhood stage:

- Affective Impressions: how children perceive their peers' emotions in group activities;

- Affective Synchrony: how accurate are children's group evaluations of emotions; and

- Empathy: the innate capacity for evaluating feelings.

While past studies have investigated the relation between movement synchrony and cooperation (Richardson, Garcia, Frank, Gergor, \& Marsh, 2012; Tuncgenc \& Cohen, 2016), this research did not identify previous attempts to measure children's affective impressions or to establish direct relations with their groupwork performance. In order to establish this relationship, we developed a series of procedures capable of evaluating cooperation and AS levels.

To evaluate AS, we have developed a scaled entitled Happiness Impression Scale - HIS. In this scale, participants are compelled to report their happiness level and evaluate the happiness level of their friends in adapted five-point Smiley Likert scales (Hall, Hume, \& Tazzyman, 2016). The HIS scale evaluates the difference between how a participant perceived the happiness level of his/her group members and the reported happiness level of these members. The lesser the difference between impression and report, the more accurate is the evaluation. If every member in a group displays good accuracy levels, the group is considered affectively synchronized. More information on the HIS scale can be seen in the methods section.

To evaluate group performance, we developed a five-day team design workshop for children aged between 7 and 14 y/o. Group design workshops are activities where children need to work together in order to develop a new product or solution to a given issue (Mechelen M. V., 2015). Group Design workshops are relatable with team building tasks where children need to exercise teamwork, dialogue, and consensus for the achievement of a common goal. Moreover, these workshops allow children to practice social skills through mediation and experimentation of different key roles in a naturalistic, self-motivating context. Due to these benefits, multiple studies are utilizing team design workshops to investigate children's social behaviors (Gibson L., 2002; Guha M. L., 2004; Mechelen M. V., 2015; Mochizuki Y., 2013).

In the proposed workshop of this research, children were divided in same-grade groups and asked to develop a new play equipment for their school field. Three activities were performed and, at the end of each, participants were compelled to fill a survey about their affective impressions of the activity and of their group members. At the end of the workshop, participants presented their ideas in group presentations which were evaluated by a school staff jury aided by design rating scales. Differences 
123 in group outcomes were observed according to individual factors such as age, gender, and grade, and

124 to the conditional variables of the activity (Task Impressions, Affective Impressions, and Affective

125 Accuracy). With the main goal of establishing a possible relation between children's affective

126 synchrony and their cooperation levels, the research worked with the following questions: Can we

127 effectively measure Affective Synchrony, and does being emotionally synchronized with group

128 members lead to better group outcomes?

\section{Methodology}

130 A design workshop entitled [removed for peer review] was performed at a school in [removed for 131 peer review] city, [removed for peer review]. In this workshop, 36 participants worked together in 132 same-grade groups to develop a new interactive playground for their school field. The workshop 133 lasted five days and was composed of three tasks. At the end of each task, participants utilized a 134 developed scale to self-report their impressions about the activity and about their group members. 135 The reports were averaged and compared with the group performance of participants, which was 136 evaluated by a jury composed of 6 school staff members. More detail on the process can be seen in 137 figure 1 and in the following subsections.

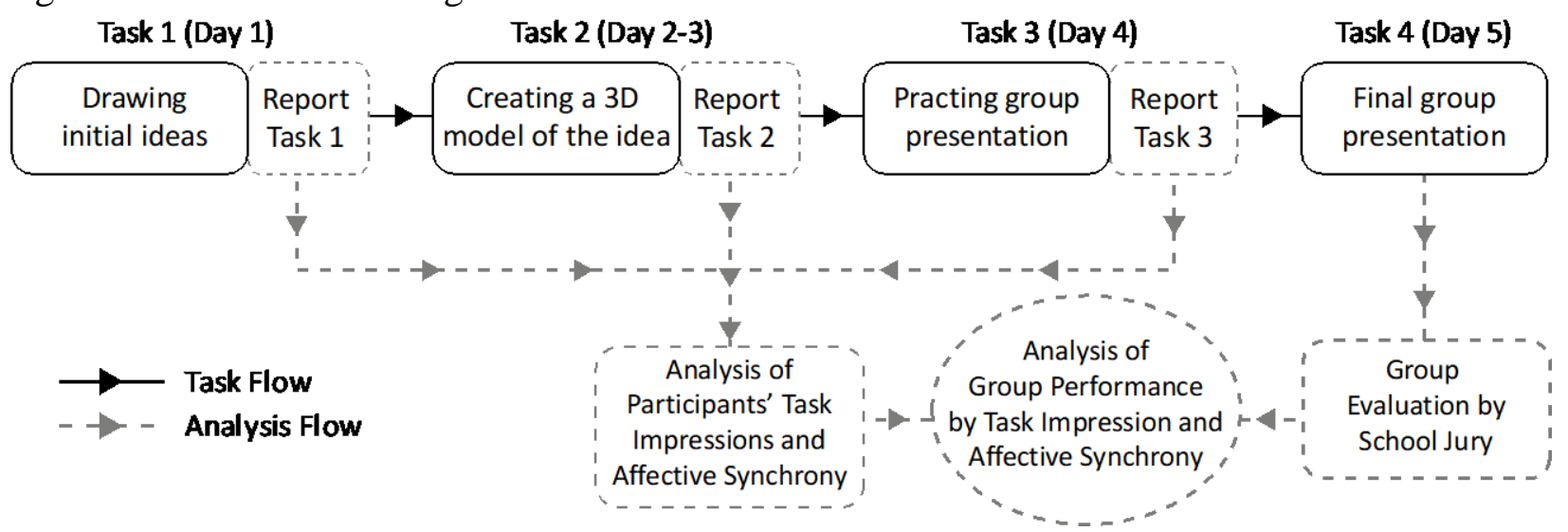

Figure 1. Flowchart displaying the performed tasks and Analysis of the study.

\subsection{Participants and Group Formation}

14036 students (15 male and 21 female) aged between 7 and 14 y/o joined the design workshop.

Participants were divided into four group sessions according to their school grade:

- Group A (2nd graders): N=9, (4 males and 5 females), Mean Age = 7, SD = 0.70;

- Group B (3rd-4th graders): N=10 (5 males and 5 females), Mean Age = 8.44, SD = 1.33;

- Group C (5th graders): N =10 (3 males and 7 females), Mean Age = 10, SD = 0.66; and

- Group D (6th-7th graders): N $=7$ (3 Males and 4 females), Mean Age = 11.85, SD = 1.46.

Asides from the final group presentation, all workshop tasks were given separately to each group. the final presentation day, or to lack/incorrect survey submission. 
2.1.1 Ethical Considerations

This research obtained approval from the Ethics Committee of the **** University. Prior to its beginning, Authorization was requested from school headmasters and from the guardians of the involved participants. Participants joined the design competition as part of the summer program activity. However, the submission of self-assessment reports needed the consent of their legal guardians. Part of a broader two-year investigation, a letter explaining the general purpose of the research was given to each guardian together with a consent request for allowing their child to become participants (opt-in). In addition, participants were constantly reassured that, if feeling unwell or with any symptoms of discomfort, they could interrupt the task at any time and restrain from responding the reports.

\subsection{Design Workshop Tasks}

The three following design tasks were performed throughout five days of the workshop:

- Sketching: Individual and group drawings of a new toy for the school field;

- Modeling: Creating a physical representation of the idea with the available materials (i.e.: Styrofoam scale base, Colored Clay, building blocks); and

- Group Presentation: Presenting the group idea with a written script and visual aids (i.e.:

All design tasks were adapted for primary school students. Participants worked in same-grade sessions, which were performed at different times throughout a running day. Each grade session lasted 90 minutes, and groups were formed according to the participants own affinity. Before the beginning of each session, an introductory presentation of the task was given. After each session, children would fill a self-report asking their impressions of the task and of their group members. In the final workshop day, the participants presented their ideas to a 6-member school staff jury, who evaluated how good was the presentation, the idea, and the teamwork.

\section{$174 \quad 2.3 \quad$ Analysis Procedures}

175 A series of tools and procedures were applied for evaluating children's groupwork, empathy pre176 disposition, task impressions, and group affective Synchrony. They are described below.

\section{$177 \quad$ 2.3.1 Pre-assessment of participant's empathy and of additional individual conditions}

178 Prior to the workshop, 22 participants had their empathy levels measured with the Kids Empathy

179 Development Scale (Reid et al., 2013). This assessment acted as a control to the affective 180 impressions and synchrony participants displayed during the workshop. Asides from empathy, the 181 individual conditions of age and gender were considered for the study evaluations.

\section{$182 \quad$ 2.3.2 Self-report Scale for task and group satisfaction}

183 Dedicate Self-assessments reports were administered three times, once at the end of each workshop 184 task. The reports were composed of 6 questions about the task and 4 about the group members 185 (supplementary material 1). Participants could answer these questions into a 5-point visual scale 186 adapted from the visual Smiley Face Scale (Hall et al., 2016). Sharing strong correlation, the results 187 of the report questions were averaged into two variables: "Task Satisfaction", and "Group

188 Satisfaction". 
In the self-report document, participants could also report the intensity of their happiness level and then evaluate each of their team member's happiness level. This was done through the developed Happiness Impression Scale - HIS (figure 2). HIS is a 5-degrees visual scale composed of five smiley faces that represent different intensity of happiness, from "not so much" to "super happy".

The HIS scale has three levels of evaluations:

- Happiness Impression: how happy participants are perceiving their group members;

- Happiness Impression Accuracy: how accurately participants are perceiving their group members happiness level; and

- Affective Synchrony: The average Happiness Impression Accuracy of all members in a group. If every participant can accurately perceive the happiness level of their group member, the group is highly synchronized.

The evaluation of individual HIA is based on the difference between the Perceived Happiness - PH that a participant has of a given team member and the Reported Happiness - RH of that member. It follows the formula below:

The closer the difference is to 0, the lesser is the difference between participant perceived Happiness and group member reported happiness. In other words, the more accurate is the participant's evaluation of their group member's happiness. In this study, we considered how participants evaluated the happiness level of their group members in each task and how accurate were these evaluations. We averaged the HIA levels of each participant in the three sessions for analysis. Although significant differences were not observed between tasks, this process allowed us to better establish differences between participants HIA levels, and ensure results were not restricted to a 212 single session.

8) How happy did You feel during the activity?

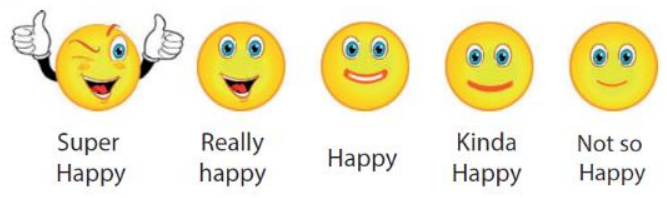

9) How happy do You think your team felt on this activity? Chose the most suitable emotion faces.

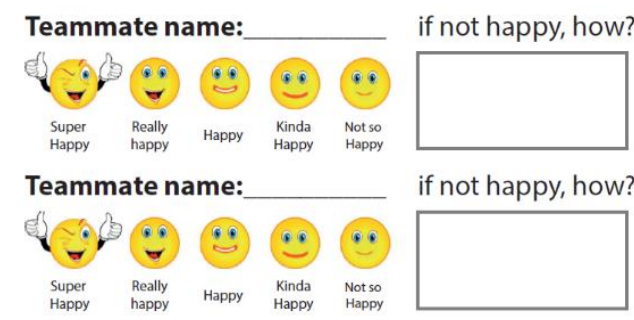

Figure 2. Happiness Impression scale questions. On the left side-figure, participants report their own happiness level. On the right-side figure, participants can evaluate the happiness level of the different members in their team. Design based on Hall et al (2016). 
Affective Synchrony Predicts the Groupwork Performance of Children

215

216

217

218

219

220

221

222

223

224

225

226

227

228

229

230

231

232

233

234

235

236

237

238

239

240

241

242

243

244

245

246

247

248

249

250

251

\subsubsection{Group Performance Evaluation through Design Score}

Participants presented their final designs in group presentations, each evaluated by a 6-member school staff evaluation jury aided with five-point design evaluation scale (supplementary material 2). The scale separately rates: how interesting and original an idea is, how clear and attractive are the designs, how viable it is, and how much effort the group has made. Results of the jury were averaged in a single group score for each presentation. Differences in group score were established in all 13 groups. After evaluating each presentation, the jury freely voted for their favorite teams in each grade group to be the winners of the workshop. Posterior analysis found that the winning teams chosen by the jury majority were also the top scorers of each grade group.

\subsection{Statistical Procedures}

linear regressions were established to evaluate age and empathy conditions on participants' reported impressions. The variable 'Gender' was evaluated by Independent-samples t-test. The sample was normalized and attended the test conditions. General linear regressions were also established to evaluate relationships between task impressions, group impressions, Happiness Impression, Happiness Accuracy, and Group Synchrony in their group design scores. Although cause and effect cannot be fully established in a correlational study, the task impressions of participants preceded their group presentations, supporting the usage of regressions.

\section{Results}

For this study analysis, we initially evaluated how the individual conditions of the participants could influence their group performance. We proceeded by observing how task impressions and Affective Synchronization levels influenced their group performance.

\subsection{Individual conditions on participant's group performance}

This study correlated the evaluated group performance of participants with three individual conditions commonly linked to group behavior: Gender; Age; and Empathy level. Figure 3 shows Boxplots and scatterplots of these analysis.

An independent-samples t-test was conducted to compare gender difference in the evaluated group performance. There was a significant difference in the scores between female $(\mathrm{M}=4.3, \mathrm{SD}=0.30)$ and male participants $(\mathrm{M}=3.9, \mathrm{SD}=0.21) ; \mathrm{t}(27)=4.12, \mathrm{p}=.0003$. These results indicate that female participants received overall better group evaluations than their male counterparts. Among the four winning groups of the workshop, three were composed of female participants.

A simple linear regression was calculated to predict the effect of age on group performance $[F(1,27)$ $=3.08, \mathrm{p}=.09 \mathrm{]}$. Age indicated to positively affect participants' evaluations up to the age of eleven $\mathrm{y} / \mathrm{o}$. Participants older than eleven tended to receive scores below average. This can be either interpreted as a lack of dedication from older students or to harsher evaluations of the jury according to age. Related to age, higher levels of empathy did not indicate significant effects on the design scores [F $(1,16)=2.42, \mathrm{p}=.15]$. While having higher rate of empathy could still have affected the participants' group work, this effect could not be directly established through a regression. 


\section{Affective Synchrony Predicts the Groupwork Performance of Children}
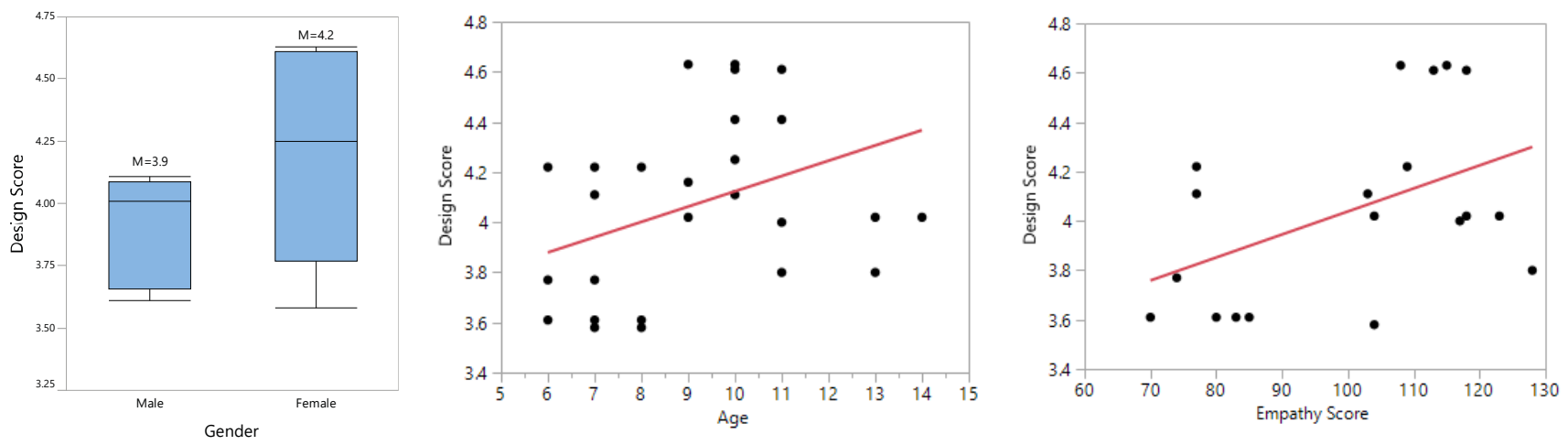

Figure 3. (Left figure) Boxplot of group performance (Design Score) by gender conditions. (center figure) Scatterplot of group performance by age. (Right figure) Scatterplot of Group Performance by Empathy Score.

253

254

255

256

257

258

259

260

261

262

\subsection{Task and Group Impressions on participant's groupwork}

We performed individual linear regressions to observe the relation of five factors with the group outcomes: reported task satisfaction (how satisfied participants were with the workshop tasks); reported team satisfaction (how satisfied participants were with their teams); Happiness Impression (How happy the participants perceived their team members to be); Happiness Impression Accuracy (how accurately participants were evaluating their team members' happiness level); and Affective Synchrony (How accurately all members from a group were evaluating each other's level of happiness). Results of the analysis can be seen in Table 1.

\section{Table 1}

Linear regression of participants reports and self-evaluations effects on their group outcomes

Significant linear regressions on design score were not established through participants' task satisfaction $[\mathrm{F}(1,22)=0.88, \mathrm{p}=.35]$, or team satisfaction $[\mathrm{F}(1,23)=0.25, \mathrm{p}=.61]$. This indicates that direct evaluations of participants' satisfaction were not enough to determine their group performance. High mean scores were observed in those reports, indicating a positive response bias. Similarly, the perceived happiness level of group members was high and did not indicate to affect group performance $[\mathrm{F}(1,25)=0.04, \mathrm{p}=.83]$. 
A significant regression with design score was established with Happiness Accuracy, $[\mathrm{F}(1,22)=$ 9.57, $\mathrm{p}=.005]$. With a $\mathrm{R} 2$ effect of 0.31 , participants who were more accurate when evaluating the happiness level of group members had better chances of receiving high group scores. A strong effect of happiness accuracy on participant's performance was also observed at group level $[\mathrm{F}(1,9)=9.89$, $\mathrm{p}=.013$ ]. With a R2 effect of 0.55 , groups in which all members shared more accurate happiness level evaluations had better chances of receiving high performance scores from the jury. Scatterplot of these analyses can be seen in Figure 4.
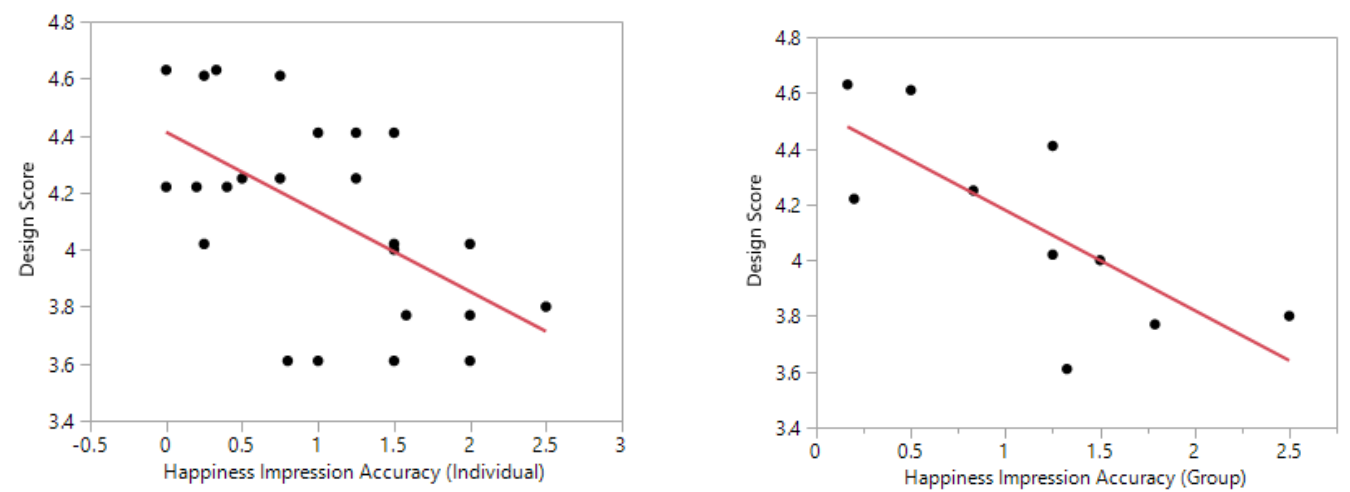

Figure 4. Scatter plots of Group Performance (design score) by participant's individual Happiness Impression Accuracy score (Left Figure) and by Group Synchrony (Right Figure).

Group Performance / Design evaluations ranged from 3.58 to 4.63 points and Affective Synchrony ranged from 2.5 to 0.165 points (with score closer to 0 indicating higher synchrony). In Group $\mathrm{A}$, the winning team scored 4.22 points in the group design evaluation and had an Affective synchrony level of 0.20 points, while the lowest performance group (3.77 points) had an Affective synchrony level of 1.79 points. In group $\mathrm{C}$ and $\mathrm{D}$, the top highest group scores (4.63 and 4.61 points) also displayed the strongest levels of Affective Synchrony (0.165 and 0.50 points), while the bottom two groups (4.00 and 3.80 points) displayed the lowest AS levels (1.5 and 2.5 points). Overall, higher group AS levels tasks led to better group performance in this study evaluation.

\subsection{Happiness Impression Accuracy and Empathy on Group Performance}

Happiness Impression Accuracy was a significant predictors of participant's group performance. The study proceeded by observing if participant's individual conditions could affect their accuracy levels. Significant differences in accuracy could be observed between gender conditions $(\mathrm{t}=-2.65, \mathrm{p}=.014)$. Overall, female participants were more accurate when evaluating their group member's levels of happiness $(\mathrm{M}=0.72, \mathrm{SD}=0.63)$ than male participants $(\mathrm{M}=1.45, \mathrm{SD}=0.59)$; This result is within expectations (Reid et al., 2013). However, a significant relation with accuracy could not be established with age, and older participants did not necessarily display higher Happiness Impression Accuracy in this study $[\mathrm{F}(1,22)=0.01, \mathrm{p}=.89]$.

Happiness Impression Accuracy is the accurate evaluations of a group member's happiness level; This, in theory, would be affected by empathy. However, a direct correlation of Accuracy with the pre-evaluated empathy score was also not established $[\mathrm{F}(1,14)=0.24, \mathrm{p}=.63]$. more than the inherent capability of being able to detect people's feelings, additional factors might be affecting the accuracy of participants during the task. In group settings, participants need to have a good capacity to evaluate the feelings of peers while actively interacting and promoting communication with them. In this context, empathy and group synchrony can be considered as complementary factors. 
300 Considering this complementary relationship, a multiple linear regression was calculated to predict

301 participant's group performance based on their Empathy and Happiness Impression Accuracy scores.

302 This multiple regression was significant $[\mathrm{F}(3,11)=12.15, \mathrm{p}=.0008]$ with an $\mathrm{R} 2$ of .76. The design

303 score of a participant is equal to 2.94 - 0.32 (Happiness Impression accuracy) + 0.013 (Empathy

304 Score), whereas Empathy Score is measured from 71 to 128 points $(M=103)$, and Happiness

305 Impression Accuracy is measured in decimals from 2.5 to 0 points $(M=1.03)$. The design score

306 increased 0.013 points for each empathy point and decreased 0.32 points for each happiness accuracy

307

308

point. When considered together, both the Empathy score $(\mathrm{P}=.001)$ and the Happiness accuracy $(\mathrm{p}=.001)$ were significant predictors of the group outcomes.

This paper investigated how the levels of Affective Impressions, Accuracy and Synchrony can influence the group performance of children. In addition, it investigated individual conditions that could influence both Affective Synchrony and Group Performance. In general, female participants were better organized than males, which might have positively affected their performance.

314 Like age, higher rates of empathy did not necessarily lead to better group performances. It is possible that having either higher or lower empathy levels than the group average could lead to instances of dissonance between participants. Further studies are necessary to investigate this hypothesis. Regardless, our current results indicate that individual empathic capacities were not sufficiently strong indicators for defining children's groupwork performance.

319 When considering the self-reported impressions, "Happiness Impression Accuracy" and "Group Synchrony" were stronger predictors for group performance than "Task" and "Group" satisfaction. High Happiness Impression Accuracy was consistent among the winning groups of the workshop and was unrelated to "how happy" these group members were perceived to be. This highly suggests that participants with high group performance were not evaluating their group members blindly, but rather sharing a state of emotional/affective synchrony, in which communication and group work could have been facilitated. Considering the potential of this variable as a predictor of group performance, we observed factors that may have led to higher affective synchrony among groups.

Following expectations, differences between participant's accuracy could be observed between gender conditions, with females displaying higher group synchrony and being better group workers than male (Reid et al., 2013). Age and Empathy, however, did not appear to be directly related to higher accuracy within children's evaluations. When considered together, Empathy and Happiness Accuracy indicated to be complementary and represented one of the main factors for defining a positive group performance. In other words, the combination of children being naturally empathic and managing to be in synchrony with their groups greatly influenced their groupwork performance.

\section{Considerations}

335 The results of this study pointed Affective Synchrony as an important and measurable indicator of children's performance in group design workshops. Moreover, rather than being directly related to empathic capabilities, AS indicated to complement it throughout children's group interactions. complementing innate empathic capabilities, AS indicates to be an important conditioner of children's cooperation in groupworks. In other words, for cooperation to occur more effortlessly, it is important to not only understand well the feelings of your team members, but also to emotionally connect with them. Considering their potential for influencing groupwork among children, Empathy and Affective Synchrony should be further investigated together in future studies. 
Among merits, this study also identified Affective Synchrony as a measurable effect among children, working towards the development of a scale that can be applicable in different group situations. The Happiness Impression Scale developed in this study indicated to be a robust measurer of children's Happiness Accuracy and Affective Synchrony. This scale can give useful insights about children's group performance in different situations. Evaluating the difference between children's impressions can counter their strong response bias when answering surveys. We are confident that this process will help us reach new understandings of children's impressions in different settings.

\subsection{Research limitations and prospects}

351 There is still much to investigate about the factors that may lead to higher or lesser Affective synchrony levels among children. As current limitations of this study, two possible unmeasured

Limitations were also observed in the current version of the HIS scale. Currently, the scale is ing on the evaluation of happiness level among participants, restricting inferences of other possible emotional states. Redesigns of the tool could consider more in-depth assessments of affective impressions within single experimental sessions.

Although group outcome evaluation followed procedures and scales of a design evaluation, the jury was composed of school staff members instead of design professionals. This fact possibly changed the focus of the evaluation. More than utilizing aesthetic and conceptual design criteria, as educators, the jury could also be evaluating the teamwork displays of the participants when selecting the winners. Thus, it remains unclear if higher levels of Affective Synchrony lead to better group designs outcomes, or to general higher levels of observable cooperation. Furthermore, our findings may be restricted to the participants and conditions of this workshop experiment. It would be beneficial to replicate this study with different samples and settings.

In conclusion, this paper contributed to the establishment of Affective Synchrony as a measurable indicator of children's group outcomes. This establishment can bring us closer to understanding the mechanisms that motivate cooperation among children. Not only that, it may also lead to the development of new procedures to nurture cooperation throughout different ages.

379 Further studies should investigate the extension of Affective Synchrony on group cooperation, as 380 well as the possible variables that can AS levels. This synchrony can be subjected to individual and situational variables. To better understand the mechanisms behind Affective Synchrony, future studies should work with experimental situations where such variables can be properly controlled. 


\section{Affective Synchrony Predicts the Groupwork Performance of Children}

\section{Acknowledgments}

385 We acknowledge [removed for blind review] school for its support throughout all research stages of this paper. [removed for blind review] School provided structure, supplies, and participants, which were all fundamental for the execution of this research.

\section{$7 \quad$ Author Contributions}

$\mathrm{RF}$, and TY contributed conception and design of the study; RF organized the database, performed the statistical analysis, and wrote the first draft of the manuscript; TY contributed to manuscript revision, read and approved the submitted version.

\section{$392 \quad 8 \quad$ Conflict of Interest}

393

The authors declare that the research was conducted in the absence of any commercial or financial relationships that could be construed as a potential conflict of interest.

\section{$9 \quad$ References}

Angerer, S., Glatzle-Rutzler, D., Lergetporer, P., \& Sutter, M. (2016). Cooperation and discrimination within and across language borders: Evidence from children in a bilingual city. European Economic Review, 90, 254-264. doi:10.1016/j.euroecorev.2016.02.022

Asher, L., \& Collins, L. M. (2012). Assessing synchrony in groups: Are you measuring what you think you are measuring? Applied Animal Behaviour Science, 138(3-4), 162-169. doi:10.1016/j.applanim.2012.02.004

Bernardi, L., Porta, C., Casucci, G., Balsamo, R., Bernardi, N. F., Fogari, R., \& Sleight, P. (2009). Dynamic Interactions Between Musical, Cardiovascular, and Cerebral Rhythms in Humans. Circulation, 119(25), 3171-3180. doi:10.1161/Circulationaha.108.806174

Branscombe, N. R., \& Wann, D. L. (1992). Role of Identification with a Group, Arousal, Categorization Processes, and Self-Esteem in Sports Spectator Aggression. Human Relations, 45(10), 1013-1033. doi:Doi 10.1177/001872679204501001

Broadhead, P. (2003). Early Years Play and Learning: developing social skills and cooperation. . London: Routledge.

Council, N. R. (1984). Development During Middle Childhood: the years from six to twelve. Washington DC: The National Academies Press.

Delaherche, E., Chetouani, M., Mahdhaoui, A., Saint-Georges, C., Viaux, S., \& Cohen, D. (2012). Interpersonal Synchrony: A Survey of Evaluation Methods across Disciplines. Ieee Transactions on Affective Computing, 3(3), 349-365. doi:10.1109/T-Affc.2012.12

Ellamil, M., Berson, J., \& Margulies, D. S. (2016). Influences on and Measures of Unintentional Group Synchrony. Frontiers in Psychology, 7. doi:ARTN 1744

\subsection{9/fpsyg.2016.01744}

Ellamil, M., Berson, J., Wong, J., Buckley, L., \& Margulies, D. S. (2016). One in the Dance: Musical Correlates of Group Synchrony in a Real-World Club Environment. Plos One, 11(10). doi:ARTN e0164783 
Ezaki, T., Horita, Y., Takezawa, M., \& Masuda, N. (2016). Reinforcement Learning Explains Conditional Cooperation and Its Moody Cousin. PLoS Comput Biol, 12(7), e1005034. doi:10.1371/journal.pcbi.1005034

Fehr, E., \& Fischbacher, U. (2004). Social norms and human cooperation. Trends in Cognitive Sciences, 8(4), 185-190. doi:10.1016/j.tics.2004.02.007

Fischbacher, U., Gachter, S., \& Fehr, E. (2001). Are people conditionally cooperative? Evidence from a public goods experiment. Economics Letters, 71(3), 397-404. doi:Doi 10.1016/S01651765(01)00394-9

Gibson L., G. P., \& Milne S. . (2002). Case Study: designing with 'Difficult' children. Paper presented at the Interaction Design and Children conference - IDC, Eindhoven: The Netherlands.

Guha M. L., D. A., Chipman G., Farber A. et al. (2004). Mixing Ideas: A New Technique for Working with Young Children as Design Partners. Paper presented at the Interaction Design with Children Conference - IDC, Maryland: USA.

Hall, L., Hume, C., \& Tazzyman, S. (2016). Five Degrees of Happiness: Effective Smiley Face Likert Scales for Evaluating with Children. Proceedings of the 15th International Conference on Interaction Design and Children (Idc2016), 311-321. doi:10.1145/2930674.2930719

Hendrix, K., van Herk, R., Verhaegh, J., \& Markopoulos, P. (2009). Increasing children's social competence through games, an exploratory study. Paper presented at the Proceedings of the 8th International Conference on Interaction Design and Children.

Hepach, R. (2017). Prosocial Arousal in Children. Child Development Perspectives, 11(1), 50-55. doi:10.1111/cdep.12209

Khalil, A. K., Minces, V., McLoughlin, G., \& Chiba, A. (2013). Group rhythmic synchrony and attention in children. Frontiers in Psychology, 4. doi:ARTN 564

\subsection{9/fpsyg.2013.00564}

Kuhn, S., Muller, B. C. N., van der Leij, A., Dijksterhuis, A., Brass, M., \& van Baaren, R. B. (2011). Neural correlates of emotional synchrony. Social Cognitive and Affective Neuroscience, 6(3), 368-374. doi:10.1093/scan/nsq044

Lergetporer, P., Angerer, S., Glätzle-Rützler, D., \& Sutter, M. (2014). Third-party punishment increases cooperation in children through (misaligned) expectations and conditional cooperation. Proceedings of the National Academy of Sciences, 201320451.

Majolo, B., \& Marechal, L. (2017). Between-group competition elicits within-group cooperation in children. Scientific Reports, 7. doi:ARTN 43277

\section{$10.1038 /$ srep43277}

Mechelen M. V., Z. B., Laenen A., \& Abeele V. V. . (2015). Challenging Group Dynamics in Participatory Design with Children. Paper presented at the 14th Interaction Design with Children Conference - IDC, Boston: Massachusetts.

Mochizuki Y., T. E., Tomisaki E., Anme T. et al. . (2013). Effects of Wood Education in a Nursery School with a focus on Changes in Children and Caregiver's Drawings. International Journal of Psychology and Behavioral Sciences, 3(6), 6. 


\section{Affective Synchrony Predicts the Groupwork Performance of Children}

462

463

464

465

466

467

468

469

470

471

472

473

474

475

476

477

478

479

480

481

482

483

484

485

486

487

488

489

490

491

492

493

494

495

496

Murata, Y., Yamaguchi, M. (2010). Education in Contemporary Japan: system and content. . Tokyo: Toshindo.

Neumann, D. L., Chan, R. C. K., Boyle, G. J., Wang, Y., \& Westbury, H. R. (2015). Measures of Empathy: Self-Report, Behavioral, and Neuroscientific Approaches. Measures of Personality and Social Psychological Constructs, 257-289. doi:10.1016/B978-0-12-386915-9.00010-3

Olson, K. R., \& Spelke, E. S. (2008). Foundations of cooperation in young children. Cognition, 108(1), 222-231. doi:10.1016/j.cognition.2007.12.003

Reid, C., Davis, H., Horlin, C., Anderson, M., Baughman, N., \& Campbell, C. (2013). The Kids' Empathic Development Scale (KEDS): A multi-dimensional measure of empathy in primary school-aged children. British Journal of Developmental Psychology, 31(2), 231-256. doi:10.1111/bjdp.12002

Richardson, M. J., Garcia, R. L., Frank, T. D., Gergor, M., \& Marsh, K. L. (2012). Measuring group synchrony: a cluster-phase method for analyzing multivariate movement time-series. Frontiers in Physiology, 3. doi:UNSP 405

10.3389/fphys.2012.00405

Roberts, L. (2018). Inclusion, Play and Empathy: Neuroaffective Development in Children's Groups. Australian and New Zealand Journal of Family Therapy, 39(1), 127-128. doi:10.1002/anzf.1288

Tong, L., Shinohara, R., Sugisawa, Y., Tanaka, E., Yato, Y., Yamakawa, N., . . Grp, J. C. s. S. (2012). Early Development of Empathy in Toddlers: Effects of Daily Parent-Child Interaction and Home-Rearing Environment. Journal of Applied Social Psychology, 42(10), 2457-2478. doi:10.1111/j.1559-1816.2012.00949.x

Tschacher, W., Rees, G. M., \& Ramseyer, F. (2014). Nonverbal synchrony and affect in dyadic interactions. Frontiers in Psychology, 5. doi:ARTN 1323

10.3389/fpsyg.2014.01323

Tuncgenc, B., \& Cohen, E. (2016). Movement Synchrony Forges Social Bonds across Group Divides. Frontiers in Psychology, 7. doi:ARTN 782

10.3389/fpsyg.2016.00782

Vanutelli, M. E., Gatti, L., Angioletti, L., \& Balconi, M. (2017). Affective Synchrony and Autonomic Coupling during Cooperation: A Hyperscanning Study. Biomed Research International. doi:Artn 3104564

\section{$10.1155 / 2017 / 3104564$}

Warneken, F. (2015). Precocious Prosociality: Why Do Young Children Help? Child Development Perspectives, 9(1), 1-6. doi:10.1111/cdep.12101 